\title{
The denoising of the TEM response from a PRBS source with Hilbert-Huang transform
}

\author{
Hai Li, Guoqiang Xue, Huasen Zhong \\ Key Laboratory of Mineral Resources \\ Institute of Geology and Geophysics, CAS \\ Beijing, China \\ thinda@163.com
}

\begin{abstract}
The denoising process is critical when processing transient electromagnetic (TEM) sounding data. For the full waveform PRBS response, an inadequate noise estimation may result in an erroneous interpretation. We consider the Hilberthuang transform and its application to suppress the noise in the PRBS response. The focus is on the thresholding scheme to weed out the energy corresponding to the noise and the analysis of the signal based on its Hilbert time-frequency representation. The method firstly decomposes the signal into the intrinsic mode function, and then, inspired by the thresholding scheme in wavelet analysis, an adaptive and interval thresholding is conducted to set to zero all the components in intrinsic mode function which are lower than a threshold related to the noise level. The algorithm is based on the characteristic of the PRBS response. The HHT based denoising scheme is tested on the synthetic data and applied to the field data with different noise level. The result shows that the proposed method has a good capability in denoising and detail preservation.
\end{abstract}

Keywords_denoising; Hilbert-huang transform; PRBS; TEM metho; EMD

\section{INTRODUCTION}

Over the past decades, transient electromagnetic method (TEM) has become widely used in hydro geophysical investigation $^{[1]}$, mineral ${ }^{[2]}$ and hydrocarbon exploration ${ }^{[3]}$. In this procedure, much effort has been made to improve the accuracy of the TEM method to meet the demands of the sensitivity properties of different exploration targets in diverse geological settings. Among this, Ziolkowski proposed to use PRBS signals as the source waveform for the EM system used in multi-transient electromagnetic method $(\mathrm{MTEM})^{[4]}$, which is considered to be a improvement over conventional source waveform .

One of the advantage of the PRBS signal is that it contains all frequencies at almost equal amplitude within a desired bandwidth ${ }^{[5]}$. In addition, the frequency range and its density are controllable by properly setting the stage and the bit duration of the PRBS signals so that the detecting accuracy for the targets at different depth can be optimized. However, the recorded full waveform PRBS response cannot be directly inverted and interpreted, and the deconvolution process is needed to obtain the impulse response which can be used to further acquire the electrical resistivity contrast underground. The deconvolution is a typical inverse problem

\author{
Pan Zhao \\ University of Chinese Academy of Sciences, Beijing, China \\ Beijing, China
}

from the Fredholm integral equation of first kind, which makes it difficult to obtain useful solution at the presence of noise. Therefore, the noise contents should be suppressed before the deconvolution procedure.

The Hilbert-Huang transform (HHT), a new signal analysis method mainly used to process the non-stationary signal, was proposed by Huang ${ }^{[6]}$. It has a vast number of diverse applications such as biomedical, watermarking and audio processing . Apart from the specific application listed above, the HHT has been proved useful in geophysical signal denoising for the seismic ${ }^{[7]}$, microseismic ${ }^{8]}$ and MT data ${ }^{[9]}$. The empirical mode decomposition (EMD) and Hilbert spectrum analysis (HSA) is naturally suited to the PRBS signal, for they share similar properties of the zero crossings and extremums. Hence, the HHT is expected to have a good effect in suppressing the noise in PRBS signal.

In this paper, the denoising scheme based on the HHT for the PRBS signal is proposed. Firstly, the principle of the HHT method and the steps involved in the decomposition and thresholding procedures are given in detail. Then, the field data for the signal with different signal to noise ratio are processed using the presented denoising scheme.

\section{THE HILBERT-HUANG TRANSFORM-BASED DENOISINGMETHOD}

The Hilbert-Huang transform consists of empirical mode decomposition and Hilbert spectral analysis. The Hilbert transform (HT) can give an easily understandable timefrequency-energy description of a time serials, while the EMD can generate components of the time serials whose Hilbert transform can lead to physically meaningful definitions of the instantaneous frequency. Therefore, the combination of HT and EMD provides a powerful signal analysis tool. In this section we will first briefly introduce the theory of the empirical mode decomposition and the Hilbert transform. Then, the principles of the adaptive and interval thresholding algorithm proposed in this paper will be presented.

\section{1) The Empirical Mode Decomposition}

The EMD is a signal decomposition algorithm proposed by Huang et al. (1998). The decomposition has a implicitly simple assumption that, at any time, the data may have many coexisting oscillatory modes of significantly different frequencies, one superimposed on the other. This mode is 
called intrinsic mode function (IMF) which is intuitive, direct and adaptive. Besides it is a posteriori defined basis derived from the data. The basis satisfies empirically all the major mathematical requirements for a time serials decomposition method, including convergence, orthogonally and uniqueness. The IMFs are found by an iterative procedure called sifting algorithm, the derived IMFs satisfy the following condition:

a) In the whole data set, the number of extremum and the number of zero crossings must equal or differ at most by one;

b) At any data point, the mean value of the envelope defined using the local minima and the envelope defined using the local maxima are zero.

Given that the PRBS satisfies these two conditions, the EMD is naturally suited to decompose the response excited by the PRBS source signal. Hence, the EMD is hopefully to get some useful decomposition outcomes in this case.

\section{2) The Hilbert Spectral Analysis}

The time serials $X(t)$ can be expressed in terms of the Hilbert amplitude and instantaneous frequency by conducting a Hilbert transform on the obtained IMFs. For each IMF $c_{j}(\mathrm{t})$, its Hilbert transform $y_{j}(t)$ is:

$$
y_{j}(t)=\frac{1}{\pi} p \int_{-\infty}^{+\infty} \frac{c_{j}(\tau)}{t-\tau} d \tau,
$$

Where $p$ is the Cauchy principle value of the singular integral. Then we can obtain the analytic function $z_{j}(t)$ with the Hilbert transform $y_{j}(t)$ of $c_{j}(\mathrm{t})$,

$$
z_{j}(t)=c_{j}(t)+i y_{j}(t)=a_{j}(t) \mathrm{e}^{i \theta(t)},
$$

Where $i=\sqrt{-1}$, and

$$
a_{j}(t)=\sqrt{c_{j}^{2}(t)+y_{j}^{2}(t)}, \theta_{j}(t)=\operatorname{arctg}\left(\frac{y_{j}(t)}{c_{j}(t)}\right) .
$$

Here, $a_{j}$ and $\theta_{j}$ is the instantaneous amplitude and phase of $j$ th order IMF respectively, and the instantaneous frequency is simply

$$
\omega_{j}(t)=\frac{d}{d t} \theta_{j}(t)
$$

Essentially, equation (2) defines the Hilbert transform as the convolution of $c_{j}(\mathrm{t})$ with $1 / t$. Therefore, it emphasizes the local properties of $c_{j}(\mathrm{t})$. The polar coordinate expression further clarifies the local nature of this expression: it is the best local fit of an amplitude and phase-varying trigonometric function to $c_{j}(\mathrm{t})$. The past application of Hilbert transform is all limited to the narrow band-passed signal, which is narrow-banded with the same number of extremum and zero crossing. It is introduced above that the IMFs are all narrow-banded and satisfy the conditions of the extremum and zero crossing. Hence, the Hilbert transform can be applied to the IMFs.
With both amplitude and frequency being a function of time, the Hilbert spectral representation for the time serials $\mathrm{X}(t)$ can be defined as:

$$
H(\omega, t)=\sum_{j=1}^{N} a_{j}(t) \exp \left(i \int \omega_{j}(t) \mathrm{d} t\right) .
$$

Thus, the marginal spectrum which represents the accumulated amplitude over that entire data span can be defined accordingly

$$
h(\omega)=\int_{0}^{T} H(\omega, t) \mathrm{d} t,
$$

Where $[0, T]$ is the temporal domain within which the data is defined. The marginal spectrum offers a measure of the total amplitude contribution from each frequency value, serving as an alternative spectrum expression to the traditional Fourier spectrum.

\section{EXAMPLES}

In this section, the methods stated previously are applied to the experimental field data. The field data are collected at molybdenum mines in Inner Mongolia, China, with the purpose of testing the equipment. The electrical dipole source and the inline receiver configuration are adopted. A 12 stage PRBS sequences with a bit rate of 512 samples per second are injected into the earth via a pair of source electrodes, the transmitting current and the inline electric field are recorded simultaneously with a sampling rate of $16 \mathrm{kHz}$. The bit rate is chosen so that the penetration depth corresponding to the frequency range of the source signal will cover the concerned depth interval. We select the recorded voltage at three different sites to exam the denoising capability of the proposed HHT method, and the voltage presented here has been normalized by the length of the source bipole and the receiver bipole.

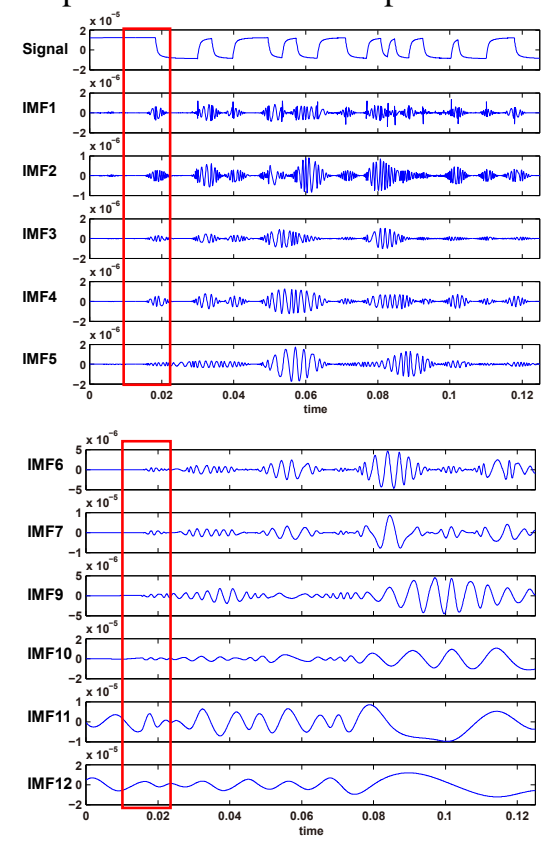

Fig. 1 The first 12 IMFs of a segment of time serials recorded at source position $=120 \mathrm{~m}$, receiver position $=580 \mathrm{~m}$ 
The analysis of the signal at this site is to demonstrate the characteristic of the response excited by the PRBS signal. Because the offset is small, the recorded signal is of good quality so that we can truly get the features of its HHT results.

Fig. 1 shows the original signal and its decomposed IMFs. It can be inferred from the Fig. that the energy of the original signal at each IMF is basically concentrated on the time interval of polarity switching for the lower order IMF. As the order of IMF gets higher, the time interval gets broadened and finally become uniformly distributed over the entire time range for the high order IMF. For instance, for the first polarity switching around $0.02 \mathrm{~s}$ in Fig. 41a, it can be seen clearly that the energy is focused on the interval around $0.02 \mathrm{~s}$ in IMF1 to IMF3. For the IMFs from IMF9 to IMF12, the concentration of energy vanished, the amplitude of IMFs is nearly in the same level. This feature can be also seen in Fig. 2a where the location of the stripes indicates the switching of polarity in the signal.

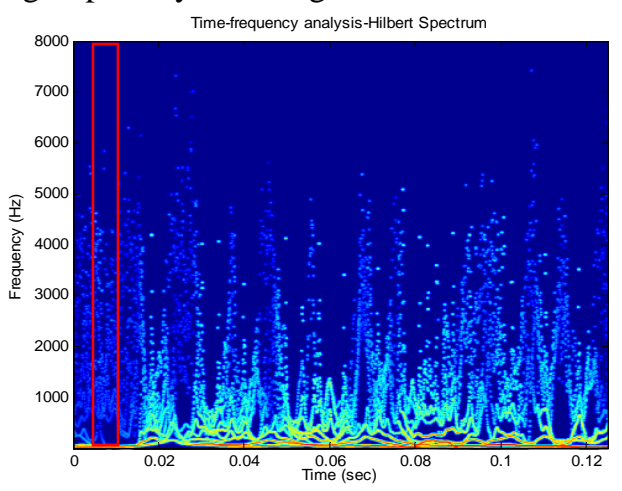

(a)

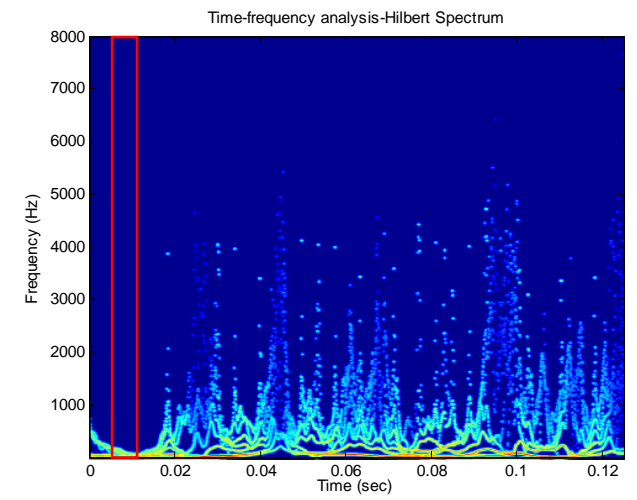

(b)

Fig. 2 HHT time-frequency energy distribution plot of a segment of time serials recorded at Source position=120 m, Receiver position=580 m (a)before and (b)after denoising

The consistency of the location of the energy concentration and the polarity switching indicates the IMFs features when the signal is of good quality. Therefore, this consistency can be used to suppress the energy from the noise by wiping out the components with relatively lower amplitude beyond the polarity switching interval. Accordingly, the proposed EMD interval thresholding with $\mathrm{C}=0.5, \mathrm{~m} 1=1$ and $\mathrm{m} 2=3$ is conducted. Fig. $2 \mathrm{~b}$ shows the
HHT time-frequency energy distribution plot of the denoised signal. The frequency component beyond the polarity switching interval has been greatly suppressed compared to the time-frequency energy distribution of the original signal in Fig. 2a. Besides, this technique has well preserved the waveform

\section{CONCLUSION}

This paper demonstrates that the HHT is a useful tool for analysis and suppression of the noise in the PRBS signal, because it distinguishes between the structured signal and noise within each IMF. The EMD decomposed the signal into different scales so that the thresholding can serve as an alternative to simple band-pass filtering with the advantage of nonstationarity, adaptivity and detail preservation. The reasoning of thresholding is to set to zero all the components in IMFs which are lower than a threshold related to the noise level. Besides, the HHT time-frequency energy distribution plot provides us with the ability to exam the signal in a instantaneous frequency aspect. The processed results of the synthetic and field data show that the denoising method presented here is highly effective in removing data defects and as much as possible retaining the useful contents in the signal.

\section{ACKNOWLEDGMENT}

This research was supported by R\&D of Key Instruments and Technologies for Deep Resources Prospecting (the National R\&D Projects for Key Scientific Instruments), Grant No. ZDYZ2012-1-05- 04.

\section{REFERENCES}

[1] Danielsen, J.E., Auken, E., Jørgensen, F., Søndergaard, V., Sørensen, K.I., 2003. The application of the transient electromagnetic method in hydrogeophysical surveys. Journal of Applied Geophysics 53, 181-198.

[2] Xue, G.Q., Qin, K.Z., Li, X., Li, G.-M., Qi, Z.-P., Zhou, N.-N., 2012. Discovery of a large-scale porphyry molybdenum deposit in Tibet through a modified TEM exploration method. Journal of Environmental \& Engineering Geophysics 17, 19-25.

[3] Streich, R., 2015. Controlled-Source Electromagnetic Approaches for Hydrocarbon Exploration and Monitoring on Land. Surveys in Geophysics, 1-34.

[4] Ziolkowski, A., Hobbs, B.A., Wright, D., 2007. Multitransient electromagnetic demonstration survey in France. Geophysics 72, F197-F209.

[5] Mutagi, R., 1996. Pseudo noise sequences for engineers. Electronics \& Communication Engineering Journal 8, 79-87.

[6] Huang, N.E., Shen, Z., Long, S.R., Wu, M.C., Shih, H.H., Zheng, Q., Yen, N.-C., Tung, C.C., Liu, H.H., 1998. The empirical mode decomposition and the Hilbert spectrum for nonlinear and nonstationary time series analysis, Proceedings of the Royal Society of London A: Mathematical, Physical and Engineering Sciences. The Royal Society, pp. 903-995. 
[7] Cai, J., 2013. A combinatorial filtering method for magnetotelluric time-series based on Hilbert-Huang transform. Explor Geophys 45, 63-73.

[8] Han, J., van der Baan, M., 2015. Microseismic and seismic denoising via ensemble empirical mode decomposition and adaptive thresholding. Geophysics 80, KS69-KS80.

[9] Baldridge, W.S., Cole, G.L., Robinson, B.A., Jiracek, G.R., 2007. Application of time-domain airborne electromagnetic induction to hydrogeologic investigations on the Pajarito Plateau, New Mexico, USA. Geophysics 72, B31-B45. 